\title{
Predisposição familiar para rotura do manguito rotador e outras tendinopatias - Um estudo de caso-controle*
}

\section{Family Predisposition for Rotator Cuff Tear and Other Tendinopathies - A Case-Control Study}

Jorge Henrique Assunção ${ }^{1}$ Breno Faria Tenrreiro ${ }^{1}$ Mauro Emilio Conforto Gracitelli ${ }^{1}$ Eduardo Angeli Malavolta ${ }^{1}$ Arnaldo Amado Ferreira Neto ${ }^{1}$

${ }^{1}$ Grupo de Ombro e Cotovelo, Instituto de Ortopedia e Traumatologia, Hospital das Clínicas, Faculdade de Medicina, Universidade de São Paulo, São Paulo, SP, Brasil
Endereço para correspondência Jorge Henrique Assunção, Rua Dr. Ovídio Pires de Campos 333, $3^{\circ}$ andar, São Paulo, SP, 05403-010, Brasil (e-mail: drjorgeassuncao@gmail.com).

Rev Bras Ortop 2020;55(4):470-475.

\section{Resumo \\ Palavras-chave \\ - manguito rotador \\ - genética \\ - tendinopatia \\ - histórico familiar \\ Objetivo Avaliar as prevalências de antecedente familiar de rotura do manguito e de tendinopatia em outras articulações em pacientes com rotura do manguito rotador e compará-las com controles pareados. Estimar a razão de chances de uma rotura do manguito rotador para estes dois fatores de risco. \\ Métodos Realizamos um estudo de caso-controle comparando pacientes submetidos ao tratamento para rotura do manguito rotador com controles assintomáticos. Todos os casos e controles foram avaliados por exames de imagem e pareados por idade $( \pm 2$ anos) e sexo. Realizamos uma entrevista utilizando um questionário padronizado, e coletamos dados referentes a vários fatores de risco. \\ Resultados Avaliamos 144 pacientes, 72 por grupo. Os pacientes com rotura do manguito rotador relataram, em maior número, a presença de familiares consanguí- neos que realizaram tratamento para a mesma doença e de lesões tendíneas em outras articulações em relação aos indivíduos controles $(p=0,005$ e $p=0,045$, respectiva- mente). Indivíduos com antecedente familiar de tratamento para rotura do manguito rotador ou com tendinopatias em outras articulações tiveram maior probabilidade de apresentar rotura do manguito rotador, com razões de chances de 3,3 (intervalo de confiança de $95 \%$ [IC95\%]=1,4-7,7) e 2,7 (IC95\%=1,1-6,9), respectivamente. \\ Conclusões Os pacientes com rotura do manguito rotador têm maior prevalência de familiares com a mesma doença e de tendinopatias ou lesões tendíneas em outras articulações. A presença de familiares consanguíneos com tratamento para rotura do manguito rotador e tendinopatias em outras articulações são fatores de risco para presença de roturas do manguito rotador.}

Trabalho desenvolvido no Grupo de Ombro e Cotovelo, Instituto de Ortopedia e Traumatologia, Hospital das Clínicas, Faculdade de Medicina, Universidade de São Paulo (HCFMUSP), São Paulo, SP, Brasil. recebido

11 de Setembro de 2018 aceito

26 de Fevereiro de 2019
DOI https://doi.org/

10.1055/s-0039-3402456. ISSN $0102-3616$.
Copyright $\odot 2020$ by Sociedade Brasileira License terms de Ortopedia e Traumatologia. Published by Thieme Revinter Publicações Ltda, Rio de Janeiro, Brazil 
Abstract

\section{Keywords}

- rotator cuff

- genetics

- tendinopathy

- family history
Objective To evaluate the prevalence of family history of rotator cuff tear and the presence of tendinopathy in other joints in patients with rotator cuff tears and to compare them with paired controls. To estimate the odds ratio for rotator cuff tear for these two risk factors.

Methods We performed a case-control study comparing patients submitted to treatment for rotator cuff tear with asymptomatic controls. All cases and controls were evaluated by imaging exams and matched by age ( \pm 2 years) and gender. We conducted an interview using a standardized questionnaire, and collected data on various risk factors.

Results We evaluated 144 patients, 72 per group. Patients with rotator cuff tears reported a higher number of consanguineous relatives who underwent treatment for the same disease and tendon injuries in other joints compared to the controls $(p=0.005$ and $p=0.045$ respectively). Individuals with a family history of treatment for rotator cuff tear or with tendinopathies in other joints were more likely to present a rotator cuff tear, with odds ratios of $3.3(95 \%$ confidence interval $[95 \% \mathrm{Cl}]=1.4-7.7$ ) and $2.7(95 \% \mathrm{Cl}=1.1-6.9)$ respectively.

Conclusions Patients with rotator cuff tear have a higher prevalence of family members with the same disease and tendinopathies or tendon injuries in other joints. The presence of consanguineous relatives with treatment for rotator cuff and tendinopathies in other joints are risk factors for the presence of rotator cuff tears.

\section{Introdução}

A tendinopatia do manguito rotador é a principal causa de dor no ombro, ${ }^{1}$ e a rotura destes tendões incide em $20 \%$ da população. $^{2} \mathrm{O}$ número de pacientes submetidos ao tratamento cirúrgico destas lesões cresce anualmente, ${ }^{3}$ e o reparo artroscópico do manguito rotador apresenta resultados clínicos satisfatórios em até $93 \%$ dos pacientes. ${ }^{4-6}$

A etiologia da rotura do manguito rotador é considerada multifatorial. ${ }^{7}$ Diminuição da vascularização tendínea, alterações degenerativas intrínsecas do tendão, micro e macrotraumatismos, síndrome do impacto, e fatores genéticos são fatores associados ao desenvolvimento das roturas do manguito rotador. ${ }^{8,9}$

Rotineiramente na prática clínica, observamos pacientes com tendinopatias ou lesões tendíneas em múltiplos sítios, e possivelmente há um componente genético envolvido na susceptibilidade do indivíduo à tendinopatia. ${ }^{10}$ Entretanto, poucos estudos avaliam a predisposição familiar ${ }^{11,12}$ ou de pacientes com lesões tendíneas em outras articulações ${ }^{13,14}$ de apresentar uma rotura do manguito rotador.

Estudos iniciais apontam que a presença de familiares com rotura do manguito rotador ${ }^{11,12}$ ou a presença de tendinopatias em outras articulações ${ }^{13,14}$ são fatores de risco para o desenvolvimento de uma rotura do manguito rotador. No entanto, a maioria dos estudos são epidemiológicos ou que utilizam banco de dados populacionais e hospitalares, ${ }^{12,14,15}$ com poucos estudos de caso-controle ${ }^{11,13}$ para avaliação desses fatores de risco. Também destacamos que a pesquisa de outros fatores de risco, como variáveis de confusão, ou pareamento por sexo e idade dos participantes raramente são feitas nos estudos de caso-controle sobre este tema.

Os objetivos do presente estudo foram avaliar as prevalências de antecedente familiar de rotura do manguito e de tendinopatia em outras articulações em pacientes com rotura do manguito rotador e compará-las com controles pareados, e estimar a razão de chances de uma rotura do manguito rotador para esses dois fatores de risco.

\section{Materiais e Métodos}

\section{Desenho do estudo, participantes e elegibilidade}

Foi realizado um estudo de caso-controle. Avaliamos pacientes com idades entre 40 e 65 anos com roturas completas do manguito rotador. 0 grupo controle foi constituído por pacientes atendidos na mesma instituição por afecções traumáticas não envolvendo a cintura escapular, sem queixa de dor nos ombros e, ao exame físico, sem a presença de sinais de rotura do manguito rotador. Todos os pacientes e controles foram submetidos a exame de imagem (ressonância magnética ou ultrassonografia) para confirmar a rotura ou a integridade do manguito rotador. Os pacientes e os controles foram pareados por idade ( \pm 2 anos) e sexo.

Os critérios de exclusão foram: doenças reumáticas, diabetes, cirurgias prévias no ombro, e processos infecciosos prévios ou atuais nesta articulação. Também não foram incluídos os pacientes com rotura do manguito rotador traumática, bem como aqueles com rotura parcial.

Este estudo foi aprovado pelo Comitê de Ética em Pesquisa da nossa instituição sob o número 11186. 


\section{Desfechos}

\section{Desfecho primário}

Foi avaliada a razão de chances de indivíduos com antecedente familiar de rotura do manguito rotador apresentarem a mesma doença, bem como a prevalência de familiares que realizaram tratamento para rotura do manguito rotador nos grupos caso e controle.

\section{Desfecho secundário}

Foi avaliada a prevalência de tendinopatia ou lesões tendíneas em outras articulações nos indivíduos com e sem rotura do manguito rotador. Avaliamos também a razão de chances de pacientes com tendinopatias em outras articulações terem uma rotura do manguito rotador em relação aos pacientes controles.

\section{Métodos de avaliação}

Realizamos uma entrevista utilizando um questionário padronizado, e coletamos os seguintes dados dos pacientes e controles: idade, sexo, raça, tabagismo, diagnóstico ou tratamento para hipertensão arterial sistêmica, hipotiroidismo, realização de esportes com os membros superiores, e atividade profissional com esforços repetitivos em abdução dos ombros (tempo maior ou igual a duas horas por dia). Fizemos também algumas questões sobre os antecedentes pessoais e familiares de roturas do manguito rotador e outras tendinopatias por meio de um questionário padronizado (-Figura $\mathbf{1}$ ).

\section{Análise estatística}

Tamanho da amostra

O cálculo da amostra utilizou um nível de significância de $5 \%$, um poder de $80 \%$, e uma razão de casos e controles de $1: 1$.

Utilizando as taxas de prevalência de rotura do manguito rotador nos familiares e controles de pacientes com a mesma doença, obtidas do estudo de Harvie et al. ${ }^{11}$ foram necessários 72 indivíduos em cada grupo.

\section{Análise dos dados}

Submetemos as variáveis contínuas à avaliação da normalidade por meio do teste de Kolmogorov-Smirnov, e da homogeneidade, por meio do teste de Levene. As variáveis contínuas foram apresentadas como médias e desvios padrão, enquanto as categóricas, como valores absolutos e percentuais. Entre as variáveis coletadas, a idade foi considerada uma variável contínua, e as demais foram consideradas variáveis categóricas.

A comparação entre os casos e controles, no que diz respeito às diferentes variáveis, foi realizada pelos testes do Qui-quadrado ou exato de Fisher para as variáveis categóricas. Para as variáveis contínuas, a comparação foi feita pelo teste $t$ de Student, se a distribuição dos dados era paramétrica, ou de Wilcoxon, se a distribuição era não paramétrica.

A diferença da prevalência de familiares consanguíneos com antecedente de tratamento para rotura do manguito rotador, bem como a presença de tendinopatia e lesões tendíneas em outras articulações nos indivíduos com rotura do manguito rotador e nos controles foi avaliada pelo teste do Qui-quadrado. Foi também calculada a probabilidade de associação destes dois fatores de risco com a rotura do manguito rotador como razão de chances, com intervalo de confiança de 95\% (IC95\%).

Utilizamos, para a análise dos dados, o programa Statistical Package for the Social Sciences (SPSS, IBM Corp., Armonk, NY, EUA), versão 21.0, e nível de significância de $5 \%$.

\section{Resultados}

Avaliamos 144 pacientes, 72 com rotura completa do manguito rotador, e 72 controles. Os casos e controles tinham média da idade de $53,6 \pm 6$ e 53,2 $\pm 6,1$, respectivamente $(p=0,592)$. Ambos os grupos foram compostos por 48 $(66,6 \%)$ mulheres e $24(33,3 \%)$ homens. Os grupos não diferiram estatisticamente em relação a raça, tabagismo e presença de hipertensão arterial sistêmica $(p=0,825 ; p$ $=0,673$; e $p=0,567$, respectivamente). As características gerais da amostra podem ser observadas na -Tabela $\mathbf{1}$.

Os pacientes com rotura do manguito rotador relataram, em maior número, a presença de familiares consanguíneos que realizaram tratamento para a mesma doença em relação aos indivíduos controles $(p=0,005)$. Indivíduos com antecedentes familiares de tratamento para rotura do manguito rotador apresentaram maior chance de ter a mesma doença, com razão de chances de 3,3 (IC95\%=1,4-7,7) (-Tabela 2). Entre os indivíduos com rotura do manguito rotador, 15 (20,8\%) mencionaram ter pelo menos 1 familiar de primeiro grau com a mesma doença, 7 (9,7\%) tinham pelo menos 1 familiar de

\section{1."Você tem ou teve algum problema ou doença nos tendões do seu corpo? Se sim, qual tendão ou articulação?" \\ 2."Você já fez alguma cirurgia em algum tendão do seu corpo, exceto o ombro? Se sim, qual tendão ou articulação?" \\ 3."Você tem algum familiar de sangue com problema ou doença nos tendões do ombro? Se sim, qual familiar ou familiares?" \\ 4. "Você tem algum familiar de sangue que fez cirurgia nos tendões do ombro? Se sim, qual familiar ou familiares?"}

Fig. 1 Questionário sobre antecedentes pessoais e familiares para rotura do manguito rotador e outras tendinopatias. 
Tabela 1 Características gerais da amostra

\begin{tabular}{|c|c|c|c|}
\hline & Casos & Controles & $\begin{array}{l}\text { Valor } \\
\text { de } p\end{array}$ \\
\hline Idade & $53,6 \pm 6$ & $53,2 \pm 6,1$ & 0,592 \\
\hline \multicolumn{4}{|l|}{ Sexo } \\
\hline Masculino & $24(33,3 \%)$ & $24(33,3 \%)$ & $>0,999$ \\
\hline Feminino & $48(66,6 \%)$ & $48(66,6 \%)$ & \\
\hline \multicolumn{4}{|l|}{ Raça } \\
\hline Branca & $48(66,6 \%)$ & $46(63,9 \%)$ & 0,825 \\
\hline Negra & $10(13,9 \%)$ & $9(12,5 \%)$ & \\
\hline Parda & $14(19,4 \%)$ & $17(23,6 \%)$ & \\
\hline \multicolumn{4}{|l|}{ Tabagismo } \\
\hline Sim & $15(20,8 \%)$ & $13(18,1 \%)$ & 0,674 \\
\hline Não & $57(79,2 \%)$ & $59(81,9 \%)$ & \\
\hline \multicolumn{4}{|c|}{ Hipertensão arterial sistêmica } \\
\hline Sim & $17(23,6 \%)$ & $20(27,8 \%)$ & 0,567 \\
\hline Não & $55(76,4 \%)$ & $52(72,2 \%)$ & \\
\hline \multicolumn{4}{|c|}{ Hipotiroidismo } \\
\hline Sim & $3(4,2 \%)$ & $4(5,6 \%)$ & 0,698 \\
\hline Não & $69(95,8 \%)$ & $68(94,4 \%)$ & \\
\hline \multicolumn{4}{|c|}{ Trabalho repetitivo com os ombros } \\
\hline Sim & $33(45,8 \%)$ & $25(34,7 \%)$ & 0,174 \\
\hline Não & $39(54,2 \%)$ & $47(65,3 \%)$ & \\
\hline \multicolumn{4}{|c|}{ Esporte com uso dos membros superiores } \\
\hline Sim & $7(9,7 \%)$ & $9(12,5 \%)$ & 0,596 \\
\hline Não & $65(90,3 \%)$ & $63(87,5 \%)$ & \\
\hline
\end{tabular}

segundo grau com diagnóstico de rotura do manguito rotador, e $1(1,4 \%)$ paciente tinha familiares de primeiro e segundo grau com a mesma doença. No total, 5 (6,9\%) controles relataram ter um familiar de primeiro de grau que realizou tratamento para rotura do manguito rotador, e $4(5,6 \%)$ tinham um familiar de segundo grau com a mesma afecção.

As tendinopatias ou lesões tendíneas em outras articulações foram mais prevalentes entre os pacientes com rotura do manguito rotador $(p=0,045)$. Estes pacientes tiveram maior probabilidade de ter uma rotura do manguito rotador,

Tabela 2 Presença de familiares consanguíneos que realizaram tratamento para rotura do manguito rotador

\begin{tabular}{|c|l|l|l|l|}
\hline & Casos & Controles & $\begin{array}{l}\text { Valor } \\
\text { de } \boldsymbol{p}\end{array}$ & $\begin{array}{l}\text { Razão de } \\
\text { chances }\end{array}$ \\
\hline & $(\boldsymbol{n}=\mathbf{7 2})$ & $(\boldsymbol{n}=\mathbf{7 2})$ & & $\begin{array}{l}\text { Intervalo } \\
\text { de confiança } \\
\text { de 95\% }\end{array}$ \\
\hline $\begin{array}{l}\text { Familiares que realizaram tratamento para rotura do manguito } \\
\text { rotador }\end{array}$ \\
\hline Sim & $23(31,9 \%)$ & $9(12,5 \%)$ & 0,005 & $3,3(1,4-7,7)$ \\
\hline Não & $49(68,1 \%)$ & $63(87,5 \%)$ & & \\
\hline
\end{tabular}

Tabela 3 Presença de tendinopatia ou lesões tendíneas em outras articulações

\begin{tabular}{|c|l|l|l|l|}
\hline & Casos & Controles & $\begin{array}{l}\text { Valor } \\
\text { de } \boldsymbol{p}\end{array}$ & $\begin{array}{l}\text { Razão de } \\
\text { chances }\end{array}$ \\
\hline & $(\boldsymbol{n}=\mathbf{7 2})$ & $(\boldsymbol{n = 7 2 )}$ & & $\begin{array}{l}\text { Intervalo } \\
\text { de confiança } \\
\text { de 95\% }\end{array}$ \\
\hline Sim & $16(22,2 \%)$ & $7(9,7 \%)$ & 0,045 & $2,7(1,1-6,9)$ \\
\hline Não & $56(77,8 \%)$ & $65(90,3 \%)$ & & \\
\hline
\end{tabular}

com razão de chances de 2,7 (IC95\%=1,1-6,9) (-Tabela 3). Entre os pacientes com rotura do manguito rotador, as tendinopatias ou lesões tendíneas mais frequentes foram a epicondilite lateral e a tendinopatia do tendão calcâneo, presentes em $8(11,1 \%)$ e $7(9,7 \%)$ dos pacientes, respectivamente.

\section{Discussão}

Nossos resultados mostram que pacientes com rotura do manguito rotador têm maior prevalência de familiares com a mesma doença $(31,9 \%)$ em relação aos indivíduos controles (12,5\%). Eles também mostram em maior número a presença de tendinopatias ou lesões tendíneas em outras articulações $(22,2 \%)$ em comparação aos indivíduos com manguito rotador íntegro (9,7\%). A presença de familiares com rotura do manguito rotador e a presença de tendinopatia ou lesões tendíneas em outras articulações foram fatores de risco para rotura do manguito rotador, com razão de chances de 3,3 e 2,7 , respectivamente.

Poucos estudos avaliam a possibilidade de familiares de indivíduos com rotura do manguito rotador terem maior risco de desenvolver a mesma doença. ${ }^{11,12}$ Harvie et al. ${ }^{11}$ afirmam que irmãos de pacientes com rotura do manguito rotador têm um risco relativo de 2,4 de apresentarem a mesma lesão, e quase 5 vezes mais risco de terem sintomas em comparação com o grupo controle. Entretanto, os autores desse estudo avaliaram apenas familiares de primeiro grau (irmãos), e utilizaram os cônjuges como controles. Devido à grande proximidade entre os casos e controles, fatores ambientais e familiares podem ter interferido na análise.

Tashjian et al. ${ }^{12}$ realizaram um estudo para avaliar um banco de dados populacional com informações de saúde do estado de Utah, Estados Unidos, e observaram que os familiares de pacientes com rotura do manguito rotador tiveram maior chance de terem a mesma doença. Familiares com grau de parentesco até o terceiro grau tiveram risco aumentado para rotura do manguito rotador, especialmente aqueles que tinham pessoas na família com esta doença abaixo dos 40 anos de idade. 0 risco relativo para rotura do manguito rotador nos familiares de segundo grau foi de 3,7, e nos de terceiro grau, de 1,8. Esse resultado e os encontrados por nós e Harvie et al. $^{11}$ sugerem uma predisposição familiar e genética para as roturas do manguito rotador. 
Para analisar a associação entre os fatores de risco e uma doença, é possível utilizar duas medidas de efeito: o risco relativo e a razão de chances. $O$ risco relativo pode ser obtido nos estudos coorte, ensaios clínicos e estudos transversais, mas não pode ser obtido diretamente nos de estudos caso-controle, apenas a razão de chances. Os estudos conduzidos por Harvie et al. ${ }^{11}$ e Tashjian et al. ${ }^{12}$ foram de coorte e retrospectivos, portanto, utilizaram o risco relativo como medida de efeito; por sua vez, o desenho do nosso estudo foi de caso-controle, $\mathrm{e}$ utilizamos a razão de chances. Em geral, quando a prevalência de uma doença na população é menor do que $10 \%$, a razão de chances tem valor muito semelhante ao risco relativo. No estudo de Yamamoto et al. ${ }^{2}$ a prevalência de rotura de manguito rotador em pacientes abaixo de 60 anos foi de $9 \%$.

Os pacientes com rotura do manguito rotador têm maior chance de apresentar tendinopatias ou lesões tendíneas em outras articulações. ${ }^{13,14}$ Tashjian et al. ${ }^{13}$ relataram que $38,7 \%$ dos pacientes com rotura do manguito rotador também indicaram tendinopatias ou lesões tendíneas em outras articulações, enquanto apenas 19,3\% dos indivíduos do grupo controle mencionaram a mesma informação. Encontramos uma prevalência menor de tendinopatias em outras articulações em ambos os grupos, mas, como esses autores, observamos uma diferença estatisticamente significativa entre os dois grupos. Fatores genéticos e familiares provavelmente estão envolvidos no desenvolvimento de lesões tendíneas em múltiplos sítios. ${ }^{13,15}$

A rotura do manguito rotador, bem como o desenvolvimento de outras lesões tendíneas, pode ser considerada multifatorial. ${ }^{7}$ Um certo número de genes podem estar envolvido na etiologia das lesões tendíneas, apresentando cada um deles uma pequena contribuição. As proteínas codificadas pelos múltiplos genes e o ambiente participam concomitantemente no desenvolvimento dessas lesões. ${ }^{16}$ Polimorfismos no gene do receptor $\beta$ relacionados ao estrogênio já foram associados à rotura do manguito rotador ${ }^{17,18} \mathrm{e}$ à tendinopatia do tendão tibial posterior. ${ }^{18}$ Genes que regulam a expressão das metaloproteinases e da tenascina $C$, enzimas importantes na homeostase tendínea, apresentam polimorfismos associados à rotura do manguito rotador, ${ }^{19,20}$ e às tendinopatias do tendão de Aquiles ${ }^{21}$ e do tendão tibial posterior. ${ }^{22,23}$ Entretanto, as funções desses genes ou de suas proteínas codificadas, na fisiopatologia das lesões tendíneas, precisam ser mais bem estudadas, e outros genes candidatos podem estar associados às lesões tendíneas.

Nosso estudo tem algumas limitações. Não avaliamos todos os fatores de risco conhecidos para a rotura do manguito rotador, como o índice de massa corpórea ${ }^{24}$ e o membro superior dominante do paciente. ${ }^{2}$ Esses fatores podem atuar como fatores de confusão nos resultados encontrados. Realizamos uma entrevista para coletar os dados sobre a presença de familiares consanguíneos com tratamento prévio para rotura do manguito rotador e sobre o tratamento atual ou prévio de tendinopatias e lesões tendíneas em outras articulações. Pacientes com rotura do manguito rotador deveriam ter melhores informações sobre as doenças ortopédicas pessoais e de seus familiares, pois a falta delas resulta em um viés de memória. E como as informações foram relatadas pelo paci- ente, os diagnósticos também podem ser imprecisos e ter aumentado a associação entre esses fatores de risco e a rotura do manguito rotador. A amostra com 144 indivíduos avaliados pode ser considerada pequena. Estudos com amostras maiores ou em outras populações devem ser realizados para confirmar a associação destes fatores de risco com a rotura do manguito rotador.

Entretanto, podemos ressaltar que nosso estudo realizou um pareamento entre casos e controles por sexo e idade com diferença máxima de dois anos. Também avaliamos outros fatores de risco que poderiam ser variáveis de confusão em nossa análise. Este refinamento metodológico não foi empregado nos poucos estudos prévios sobre o tema. Também realizamos exames de imagem para avaliar a rotura ou integridade do manguito rotador em todos participantes do estudo. Apenas no estudo conduzido por Harvie et al. ${ }^{11}$ também foram feitos exames de imagem nos casos e controles, e estudos prévios ${ }^{25,26}$ mostram que até $60 \%$ dos pacientes com rotura do manguito rotador podem ser assintomáticos. Também podemos afirmar que nossos achados colaboram na identificação de importantes fatores de risco para o desenvolvimento das roturas do manguito rotador, e enfatizam a necessidade de mais pesquisas para identificação de marcadores genéticos para o desenvolvimento de lesões tendíneas, possibilitando a identificação de indivíduos suscetíveis.

\section{Conclusões}

Os pacientes com rotura do manguito rotador têm maior prevalência de familiares com a mesma doença e de tendinopatias ou lesões tendíneas em outras articulações. A presença de familiares consanguíneos com tratamento para rotura do manguito rotador e tendinopatias em outras articulações são fatores de risco para a presença de roturas do manguito rotador.

\section{Conflito de Interesses}

Os autores não tem nenhum a declarar.

\section{Referências}

1 Mitchell C, Adebajo A, Hay E, Carr A. Shoulder pain: diagnosis and management in primary care. BMJ 2005;331(7525):1124-1128

2 Yamamoto A, Takagishi K, Osawa T, et al. Prevalence and risk factors of a rotator cuff tear in the general population. J Shoulder Elbow Surg 2010;19(01):116-120

3 Malavolta EA, Assunção JH, Beraldo RA, Pinto GMR, Gracitelli MEC, Ferreira Neto AA. Rotator cuff repair in the Brazilian Unified Health System: Brazilian trends from 2003 to 2015. Rev Bras Ortop 2017;52(04):501-505

4 Checchia SL, Doneux S, Miyazaki AN, et al. Avaliação dos resultados obtidos na reparação artroscópica das lesões do manguito rotador. Rev Bras Ortop 2005;40(05):229-238

5 Godinho GG, França FO, Freitas JMA, et al. Avaliação da integridade anatômica por exame de ultrassom e funcional pelo índice de Constant \& Murley do manguito rotador após reparo artroscópico. Rev Bras Ortop 2010;45(02):174-180

6 Antonio M, Veado C, Afonso I, Filho A, Duarte RG, Leitão I. Avaliação funcional do reparo artroscópico das lesões completas do manguito rotador associado a acromioplastia. Rev Bras Ortop 2008;43(11/12):505-512 
7 Longo UG, Berton A, Khan WS, Maffulli N, Denaro V. Histopathology of rotator cuff tears. Sports Med Arthrosc Rev 2011;19(03):227-236

8 Maffulli N, Longo UG, Berton A, Loppini M, Denaro V. Biological factors in the pathogenesis of rotator cuff tears. Sports Med Arthrosc Rev 2011;19(03):194-201

9 DE Giorgi S, Saracino M, Castagna A. Degenerative disease in rotator cuff tears: what are the biochemical and histological changes? Joints 2014;2(01):26-28

10 Magra M, Maffulli N. Genetic aspects of tendinopathy. J Sci Med Sport 2008;11(03):243-247

11 Harvie P, Ostlere SJ, Teh J, et al. Genetic influences in the aetiology of tears of the rotator cuff. Sibling risk of a full-thickness tear. J Bone Joint Surg Br 2004;86(05):696-700

12 Tashjian RZ, Farnham JM, Albright FS, Teerlink CC, CannonAlbright LA. Evidence for an inherited predisposition contributing to the risk for rotator cuff disease. J Bone Joint Surg Am 2009;91 (05):1136-1142

13 Tashjian RZ, Saltzman EG, Granger EK, Hung M. Incidence of familial tendon dysfunction in patients with full-thickness rotator cuff tears. Open Access J Sports Med 2014;5:137-141

14 Titchener AG, White JJ, Hinchliffe SR, Tambe AA, Hubbard RB, Clark DI. Comorbidities in rotator cuff disease: a case-control study. J Shoulder Elbow Surg 2014;23(09):1282-1288

15 Tashjian RZ, Farnham JM, Granger EK, Teerlink CC, Cannon-Albright LA. Evidence for an Environmental and Inherited Predisposition Contributing to the Risk for Global Tendinopathies or Compression Neuropathies in Patients With Rotator Cuff Tears. Orthop J Sports Med 2016;4(04):2325967116642173

16 September AV, Schwellnus MP, Collins M. Tendon and ligament injuries: the genetic component. Br J Sports Med 2007;41(04): 241-246, discussion 246

17 Motta GdaR, Amaral MV, Rezende E, et al. Evidence of genetic variations associated with rotator cuff disease. J Shoulder Elbow Surg 2014;23(02):227-235
18 Teerlink CC, Cannon-Albright LA, Tashjian RZ. Significant association of full-thickness rotator cuff tears and estrogen-related receptor- $\beta$ (ESRRB). J Shoulder Elbow Surg 2015;24(02):e31-e35

19 Assunção JH, Godoy-Santos AL, Dos Santos MCLG, Malavolta EA, Gracitelli MEC, Ferreira Neto AA. Matrix Metalloproteases 1 and 3 Promoter Gene Polymorphism Is Associated With Rotator Cuff Tear. Clin Orthop Relat Res 2017;475(07):1904-1910

20 Kluger R, Burgstaller J, Vogl C, Brem G, Skultety M, Mueller S. Candidate gene approach identifies six SNPs in tenascin-C (TNC) associated with degenerative rotator cuff tears. J Orthop Res 2017; 35(04):894-901

21 Mokone GG, Gajjar M, September AV, et al. The guanine-thymine dinucleotide repeat polymorphism within the tenascin- $C$ gene is associated with achilles tendon injuries. Am J Sports Med 2005;33 (07):1016-1021

22 Godoy-Santos A, Cunha MV, Ortiz RT, Fernandes TD, Mattar RJr, dos Santos MCLG. MMP-1 promoter polymorphism is associated with primary tendinopathy of the posterior tibial tendon. J Orthop Res 2013;31(07):1103-1107

23 Saunders CJ, van der Merwe L, Posthumus M, et al. Investigation of variants within the COL27A1 and TNC genes and Achilles tendinopathy in two populations. J Orthop Res 2013;31(04): 632-637

24 Gumina S, Candela V, Passaretti D, et al. The association between body fat and rotator cuff tear: the influence on rotator cuff tear sizes. J Shoulder Elbow Surg 2014;23(11):1669-1674

25 Yamamoto A, Takagishi K, Kobayashi T, Shitara H, Osawa T. Factors involved in the presence of symptoms associated with rotator cuff tears: a comparison of asymptomatic and symptomatic rotator cuff tears in the general population. J Shoulder Elbow Surg 2011; 20(07):1133-1137

26 Tempelhof S, Rupp S, Seil R. Age-related prevalence of rotator cuff tears in asymptomatic shoulders. J Shoulder Elbow Surg 1999;8 (04):296-299 